\title{
Microstructures of Surface Alloyed High Manganese Cast Steels
}

$$
\text { Chang-Qing GUO }{ }^{1, ~ a ~} \text {, An-Jun SHI }{ }^{2, b} \text {, Ji-Wei HAN }{ }^{3, c}
$$

1,2,3 School of Materials Science \& Engineering, Jiangsu University, Zhenjiang, Jiangsu, 212013,

China

\author{
aguo_changqing@126.com, b947227392@qq.com, ${ }^{\mathrm{c}} 15751010394 @ 163 . c o m$
}

Keyword: Ni-WC powders, High manganese cast steel, V-EPC, Microstructures, Surface alloying.

\begin{abstract}
EPS templates attaching a compressed powder disk on a surface that are mainly consisted of Ni-WC powders were prepared. High manganese molten steels were poured into the templates forming a surface alloyed sample using V-EPC process. After water quenching from $1050^{\circ} \mathrm{C}$, the microstructures of the steels were observed and analyzed using optical microscope, SEM and EDS. Macrostructural observation exhibits that the surface alloyed layer is dense and there are no obvious defects. Microstructural investigation shows that the steels from surface to core are consisted of two different parts, the surface alloyed layer and matrix. There are no any retained Ni-WC particles in the surface alloyed layer and the microstructures within this layer are composed of a small amount of fish-bone-like tungsten-rich fine carbides, a large amount of interdendritic carbides and dendritic austenite. Small amounts of carbide particles and net carbides at the grain boundaries in the matrix near the surface alloyed layer can be observed.
\end{abstract}

\section{Introduction}

High manganese cast steels are the traditional wear-resistant materials. Normally, the wear resistance can be exhibited when the materials are imposed by the effect of high stress, which led to induce the formation of high hardness of martensite on working surface. However, the steel components often work at the conditions with low or medium stress, which result in increasing surface hardness limitedly, from HB180-240 to HB450-550[1]. Thus, the wear resistance of high manganese cast steel can not be fully utilized in a normal service condition. To solve this problem, researchers attempted methods strengthening the steel so as to increase the hardness of martensite. These methods can be classified two types. The one is self-strengthening, including solution, grain refinement and dispersing strengthening by the addition of certain amounts of alloying elements, such as $\mathrm{Cr}, \mathrm{Mo}, \mathrm{V}$ and $\mathrm{Ni}$ dissolving in the lattice of $\mathrm{Fe}$, or fining the matrix grain by the formation of $\mathrm{TiC}$ and $\mathrm{NbC}$ carbides, or precipitating large amounts of fine and dispersing carbide particles in matrix. Trial results demonstrate that these measures are useful, but have unremarkable effects [2]. The other is the surface hardening, which involves fabricating a surface alloying layer that contains large amounts of hard carbides [3-5]. Trial results showed that this type of measure is effective and enable to significantly increase the service life of the components [6]. Therefore, the latter one is a promising way and should develop as an important direction.

WC particles have high hardness (HV2000-3000), high wear resistance, high elastic modulus and chemical stability. Moreover, the particles have strong wettiability with molten steels (wet angle $\theta \approx 0$ ) [7]. Thus, it is one of the most commonly used reinforcement particles in iron and steel matrices composites. This paper intends to use Ni based-WC particles as the reinforcement phases because the particles have stronger wettiability with molten steels and V-EPC (vacuum expandable pattern casting) as the casting process because it can fully exhaust the gas or moisture generated during casting. The results could be used in future to fabricate relevant composites.

\section{Experimental Materials and Methods}

The type of Ni-65WC carbide particles with size of 15-30um were used as the reinforcement 
phases, which contained about $65 \mathrm{wt} \% \mathrm{WC}$ and were made by Beijing Iron and Steel General Research Institute. Table 1 lists the chemical composition of the particles. In addition, high carbon ferro-chromium alloy particles with size less than $30 \mathrm{um}$ that contain $6.8 \mathrm{wt} \% \mathrm{C}$ and $67.5 \mathrm{wt} \% \mathrm{Cr}$ were also used to adjust the proportion of $\mathrm{Ni}-\mathrm{WC}$ particles and increase the volume fraction of carbide particles in the surface alloyed layer. The ferro-chromium alloy particles were obtained by crushing large alloy lumps into small pieces and then grinding the pieces into powders.

Tab. 1 The chemical composition of Ni-WC particles (wt \%)

\begin{tabular}{cccc}
\hline & $\mathrm{Ni}$ & WC & others \\
\hline content & 35 & 65 & 5 \\
\hline
\end{tabular}

First step for the fabrication of a surface alloying layer was the preparation of $\mathrm{Ni}-\mathrm{WC}$ particle containing disks. The disks were consisted of 50vol.\%Ni-WC particles, 40vol.\% high carbon ferrochromium alloy powders, 5vol.\% boric acid powders and 5vol.\% EPS (expanded polystyrene spheres) powders. The addition of EPS powders aims to improve the infiltration of liquid steels into disks. These powders were weighted and then put into a small laboratory grinding machine for mixing about 20 minutes with the ratio of powder to grinding ball 10 to 1 . The powders were then added about $3 \mathrm{vol} \%$ organic adhesive and a small amount of ethanol for wetting. The wetting powders were compressed into disks with size of $\Phi 20 \mathrm{~mm} \times 5 \mathrm{~mm}$ using a special mold. The disk was then attached on a surface of $25 \mathrm{~mm} \times 25 \mathrm{~mm} \times 100 \mathrm{~mm}$ EPS template. The whole EPS template was brushed a layer of anti-adhesive sand coating with thickness of $1-2 \mathrm{~mm}$, which is mainly composed of $\mathrm{MgO}$ powders, and then baked at $50^{\circ} \mathrm{C}$ for 3 to 5 hours for driving out of moisture.

Second step was put the EPS templates into a large steel box. Granular quartzite sands were then filled into the box. Finally, a plastic film was used to cover at the top surface of the box for sealing.

High manganese cast steel was melted in a $50 \mathrm{Kg}$ medium frequency induction furnace using a crucible lined by magnesia particles. When temperature of the molten steels were reached at about $1550^{\circ} \mathrm{C}, 0.15 \mathrm{wt} \%$ aluminum was added for de-oxidation. The molten steels were then poured into the EPS template while the vacuum pump was switched on. After cooling to room temperature, the box was upset down and the castings were taken out. The metallographic samples were gained from the interim part of the castings after austenitizing at $1050^{\circ} \mathrm{C}$ for 30 minutes and then quenching in water.

Microstructural observation and analysis were employed the OYMPUS-AX700 optical microscopy and Quanta 4000 scanning electron microscopy.

\section{Experimental Results}

Figure 1 exhibits an optical microstructure of a sample with a surface alloyed layer. It can be seen that the upper alloyed layer appears large amounts of dendritic matrix and interdendritic carbides, while the microstructures in matrix are similar to the normal morphology of high manganese cast steels. From a magnified microstructure as shown in Figure 2(a), it can be found that the interdendritic carbides in the alloyed layer are actually consisted of two types, the one appearing large black blocks that are dominate in the carbides, the other being fine white fish-bone-like blocks. EDS tests show that the large black carbides contain large amounts of Fe and certain amounts of $\mathrm{Mn}, \mathrm{Cr}, \mathrm{Ni}$ and $\mathrm{W}$, the white fish-bone-like fine carbides are rich in $\mathrm{W}$ and contain a small amount of $\mathrm{Fe}, \mathrm{Mn}, \mathrm{Cr}$ and $\mathrm{Ni}$, as shown in Figure 2 (b) and (d). Thus, it can be expected that the large black ones might be M7C3 or M3C carbides and the fine white ones be WC or W2C carbides. XRD tests confirm the expectation, as shown in Figure 3. Moreover, it shows that the matrix in the alloyed layer is also austenitic. However, it can not be found any Ni-WC particles in the surface alloyed layer, indicating that the carbide particles are totally decomposed during infiltration. 


\section{Discussion}

When the high manganese molten steel is poured into the V-EPC system, the EPS templates are quickly vaporized under the effect of high temperature. The gas generated from this vaporization is then elicited from the disk, anti-adhesive sand coating and the gap of sand particles via tubs buried in the steel box. The left space is then filled with the molten steel. When the liquid steel meets with the disk, it begins to infiltrate in it and consequently, $\mathrm{Ni}-\mathrm{WC}$ particles and high carbon ferrochromium alloy powders gradually decomposed or dissolved. Ni-WC particles have a melting point of about $1200^{\circ} \mathrm{C}$, so the particles can be decomposed under the effect of molten steel. However, the decomposition can be further prompted by the iron melt as the balanceable melting point of the particle interface with the steel is significantly lowed due to uneven surface of the particles [8]. High carbon ferrochromium alloy particles have melting point of about $1340^{\circ} \mathrm{C}$ [9]. Thus, the particles can be also liquated during liquid steel infiltration. The decomposition of $\mathrm{Ni}-\mathrm{WC}$,i.e., $\mathrm{Ni}-\mathrm{WC} \rightarrow[\mathrm{Ni}]+[\mathrm{W}]+[\mathrm{C}]$ and dissolution of the high carbon ferrochromium alloy powders let the melt in surface alloying layer being rich in $\mathrm{C}, \mathrm{Ni}, \mathrm{W}$ and $\mathrm{Cr}$, but poor in $\mathrm{Fe}$ and $\mathrm{Mn}$. However, the constituents in the steel matrix are totally different, to be rich in Fe and $\mathrm{Mn}$, but poor in $\mathrm{C}, \mathrm{Ni}, \mathrm{W}$ and $\mathrm{Cr}$. Consequently, mutual diffusion of the alloy elements between the two parts occurred, with $\mathrm{Ni}, \mathrm{C}, \mathrm{W}$ and $\mathrm{Cr}$ tending to diffuse from surface layer to matrix, while $\mathrm{Fe}$ and $\mathrm{Mn}$ from matrix to the surface layer. When melt temperature in surface layer is lowered to its melting point, hypoeutectic austenite begin to precipitate from the melt. When the melt in the surface alloying layer was further cooled to eutectic temperature, eutectic reaction is taken place, resulting in the formation of eutectic austenite and carbides, i.e. $\mathrm{L} \rightarrow \mathrm{r}+\mathrm{MxCy}$. Because the surface melt contains elements of $\mathrm{Ni}, \mathrm{W}, \mathrm{Mn}$ and $\mathrm{Cr}$, certain amounts of these alloying elements should dissolve in the $\mathrm{MxCy}$ carbides. This is the reason why the carbides contain elements $\mathrm{Ni}, \mathrm{W}, \mathrm{Mn}$ and $\mathrm{Cr}$. During subsequent cooling, supersaturated alloying elements of $\mathrm{C}, \mathrm{W}, \mathrm{Mn}$ and $\mathrm{Cr}$ in austenite either precipitating as new carbides or let the eutectic carbides growing or continuously diffusing to matrix zone.

$\mathrm{W}$ atom has larger nuclei radius than that of $\mathrm{Fe}$, so this atom has a strong tendency to segregate at the grain or phase boundaries. When $\mathrm{W}$ and $\mathrm{C}$ atoms segregated at grain or phase boundaries were accumulated certain amounts, a reaction $\mathrm{W}+\mathrm{C} \rightarrow \mathrm{WC}$ or $\mathrm{W}+\mathrm{C} \rightarrow \mathrm{W} 2 \mathrm{C}$, is taken place. This might be the process of the formation of the fine $\mathrm{W}$ rich fish-bone-like carbides in the surface alloyed layer.

$\mathrm{C}, \mathrm{W}$ and $\mathrm{Cr}$ contents in the matrix at the vicinity of surface alloyed layer are higher than the matrix owing to the diffusion of these alloying elements from the layer and supersaturated elements tended to form different types of carbides. Therefore, small amounts of carbide particles in the matrix and net carbides at the grain boundaries can be found in microstructure.

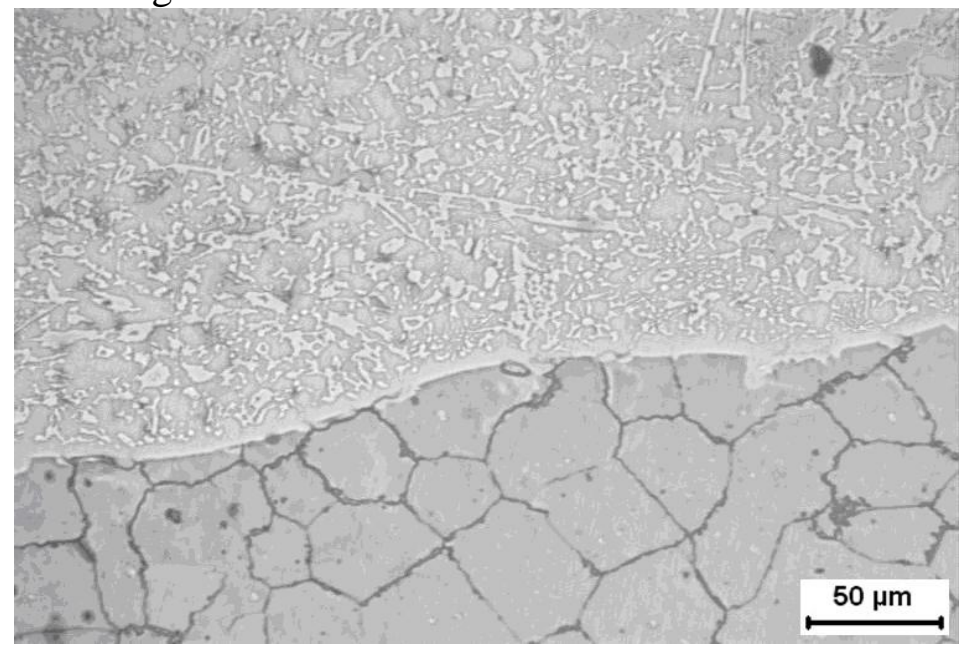

Fig. 1 Optical microstructures of the Ni-WC surface alloyed high manganese cast steel. 

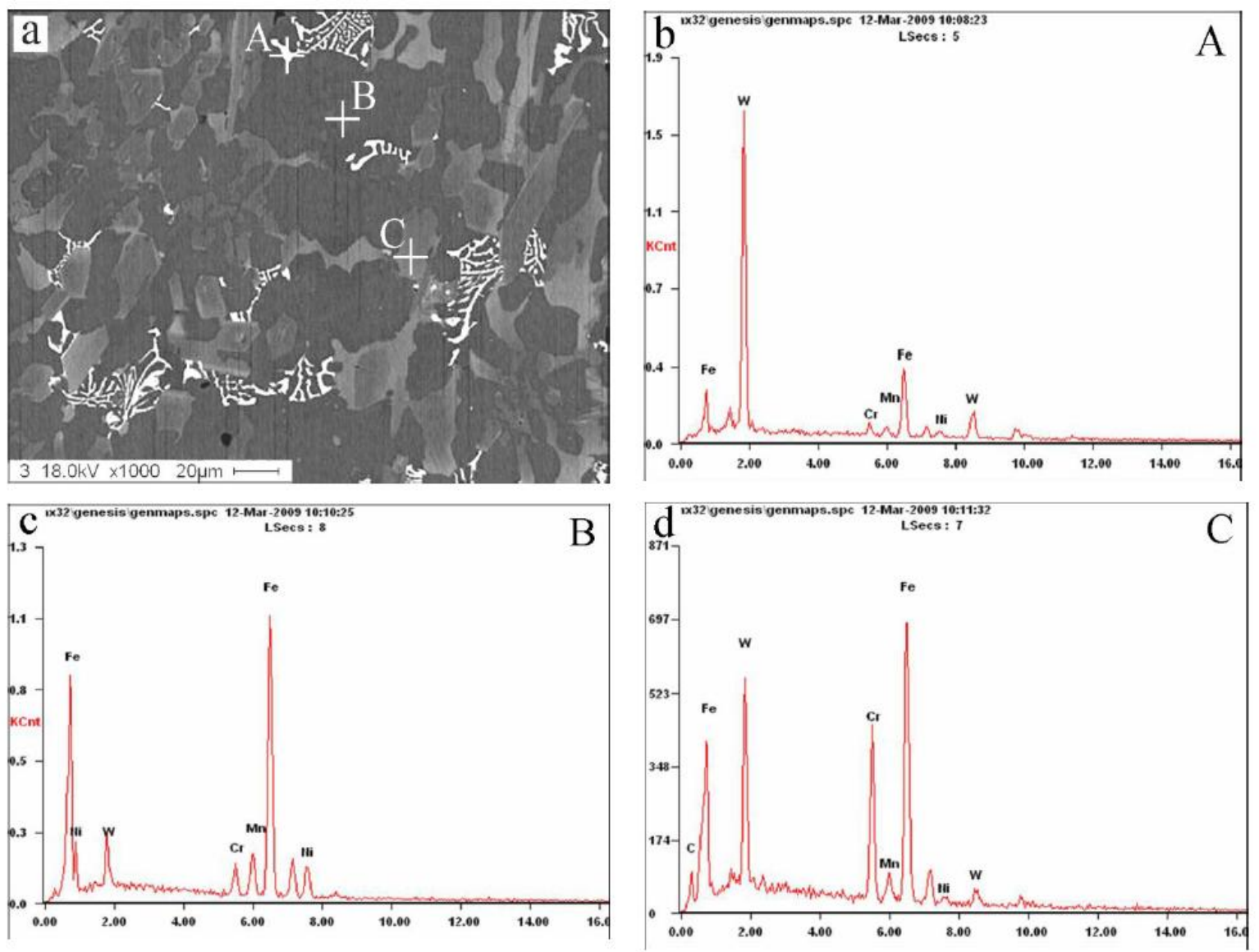

Fig. 2 Microstructures and EDS analysis of the surface alloyed layer

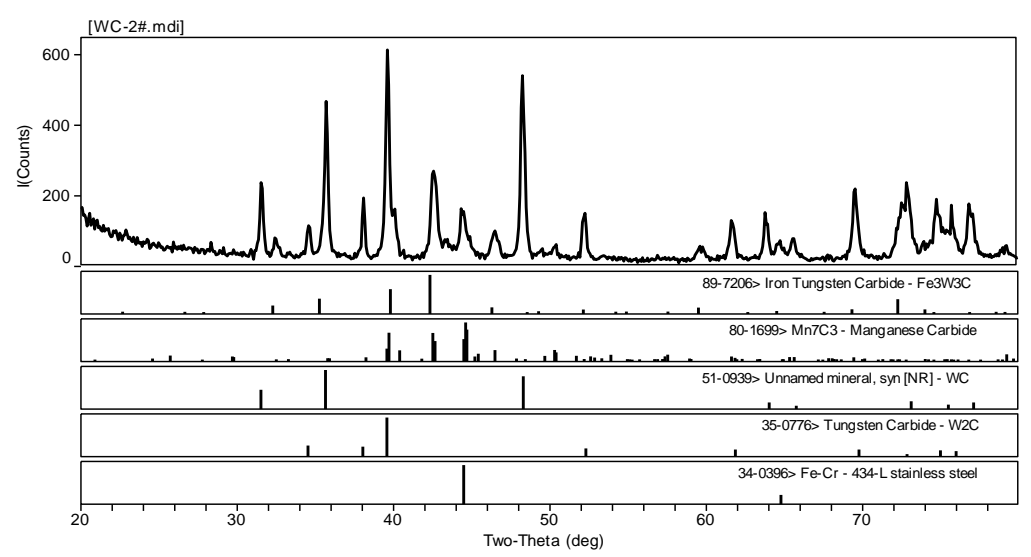

Fig. 3 XRD test result for the surface alloyed layer

\section{Conclusions}

(1) Ni-WC particles in the surface alloyed layer are totally decomposed during infiltration;

(2) The microstructures of Ni-WC surface alloyed high manganese cast steels are fallen into two different parts, the alloyed surface layer and matrix. The microstructures in the surface alloyed layer are consisted of fine $\mathrm{WC}+\mathrm{W} 2 \mathrm{C}$ carbides, a large amount of $\mathrm{MxCy}$ carbides and dendritic matrix;

(3) There are not obvious microstructural defects in the surface alloyed layer. 


\section{Acknowledgements}

The author would like to acknowledge a financial support provided by senior talent fund project of Jiangsu University under grant No. 12JDG033.

\section{References}

[1] R. B. Wang, and S. Xue, Mechanical Engineering. 31, 6(1986).

[2] Z. Z. Zhang, Editor, Metallurgical Industry Press, Beijing (2002).

[3] C. H. Ji, and C. J. Zhang, Foundry. 130, 49(2000).

[4] J. Cheng, and C. Q. Guo, J. of Inner Mongolia University of Science \& Technology. 211, 26 (2007).

[5] F. X. Zhang, Metal Hotworking Technology. 29, 6 (1998).

[6] H. J. Song, and G. S. Zhang, Foundry Technology. 4684, 26 (2005).

[7] G. S. Zhang, G. Y. Liu, J. D. Xing, Y. M. Gao, and J. B. Chen, J. OF XIAN JIAOTONG UNIVERSITY. 757, 39(2005).

[8] X. Q., You, and H. Ren, Foundry. 1063, 26(2003).

[9]H. Qi, WC particles reinforced iron and steel matrices selected area composites and its application. Kunming University of Science and Technology (2001). 